\title{
Sistema automatizado de monitoreo de parámetros físico-químicos en producción de alevines Gamitana (Colossoma macropomum)
}

\section{Automated monitoring system of physicochemical parameters in production of Gamitana fingerlings (Colossoma macropomum)}

\author{
García-Castro, Juan [D 0000-0002-8890-8800]।; Ascón-Dionicio, Gilberto [D 0000-0002-5554-4262]। \\ 'Universidad Nacional de San Martín, Tarapoto, Perú \\ $\bowtie$ jcgarcia@unsm.edu.pe \\ Recibido: 29/10/2021; \\ Aceptado: 30/II/2021; \\ Publicado: 20/01/2022
}

Resumen: La acuicultura es fuente de producción de alimentos con alto valor nutricional, además de ser una actividad económica generadora de empleo e ingresos; en Perú es una alternativa importante para el desarrollo económico y social, sin embargo, es poco tecnificada. Frente a ello, el propósito fue disminuir la tasa de mortalidad de Gamitana durante los diez primeros días de vida en el proceso de reproducción artificial con la implementación de un sistema de control de los parámetros físico-químicos del agua en el laboratorio de una estación pesquera. Para tal efecto, se empleó un estudio aplicado de desarrollo tecnológico dividió en tres componentes: I) análisis del proceso de reproducción artificial del Colossoma macropomum; 2) diseño e implementación de un sistema de medición y control de los parámetros físicoquímicos del agua en el laboratorio de reproducción artificial y 3) medición de los parámetros físicos químicos del agua y la tasa de mortalidad de en los diez primeros días de vida del Colossoma macropomum con y sin sistema automatizado de control. Obteniéndose análisis de los procesos productivos, para luego diseñar el sistema de control de los parámetros del agua con el uso de sensores y microcontroladores (arduino), lográndose así disminuir la tasa de mortalidad de los alevines en un $21.7 \%$.

Palabras clave: mortalidad; parámetros del agua; reproducción artificial; sistema de control

Abstract: Aquaculture is a source of food production with high nutritional value, in addition to being an economic activity that generates employment and income; In Peru, it is an important alternative for economic and social development, however, it is low-tech. Against this, the purpose was to reduce the mortality rate of Gamitana during the first ten days of life in the artificial reproduction process with the implementation of a control system of the physico-chemical parameters of the water in the laboratory of a fishing station. . For this purpose, an applied study of technological development was used divided into three components: I) analysis of the artificial reproduction process of Colossoma macropomum; 2) design and implementation of a system for measuring and controlling the physical-chemical parameters of water in the artificial reproduction laboratory and 3) measurement of the physical-chemical parameters of water and the mortality rate in the first ten days of life Colossoma macropomum with and without automated control system. Obtaining analysis of the production processes, to then design the control system of the water parameters with the use of sensors and microcontrollers (Arduino), thus reducing the mortality rate of the fingerlings by $21.7 \%$.

Keywords: mortality; water parameters; artificial reproduction; control system

Cómo citar / Citation: García-Castro, J. \& Ascón-Dionicio, G. (2022). Sistema automatizado de monitoreo de parámetros físico-químicos en producción de alevines Gamitana (Colossoma macropomum). Revista agrotecnológica amazónica, 2(I), e240. https://doi.org/I0.5I252/raa.v2il.240 


\section{Introducción}

La acuicultura a nivel mundial tiene una importante relevancia, debido a que es una fuente de producción de alimento de alto valor nutricional por lo que se ha convertido en una actividad económica generadora de empleo e ingresos (Sánchez Calle et al., 202I). La acuicultura continental de peces de escama, el tipo de operación acuícola más habitual en el mundo, supuso el $65 \%$ del incremento de la producción pesquera en el período 2005-20 I4. El cultivo continental de peces de escama en estanques de tierra es, con mucho, la práctica acuícola que más contribuye a la seguridad alimentaria y la nutrición en los países en desarrollo (FAO, 20I6).

En ese contexto, para la Región San Martín - Perú, la acuicultura se ha convertido en una alternativa para el desarrollo económico y social de la población en el marco de las políticas regionales, permitiendo la generación de empleo y el incremento de los niveles de ingresos; además genera oportunidades para mejorar la calidad de vida y puede integrarse con otras actividades como la agricultura; sin embargo, independiente de quien lo practique (grandes, medianos o pequeños productores), la acuicultura promueve la inclusión económica y social de manera sostenible (DIREPRO, 20I4).

La Gamitana es el pez más representativo entre los peces escamosos del río Amazonas y es muy demandado por su carne, teniendo una mayor importancia económica entre las especies del género Colossoma, aportando la proteína animal a los habitantes de la Amazonía peruana (FONDEPES, 2004).

De acuerdo con Alvan-Aguilar et al. (2021), la acuicultura es el cultivo de organismos acuáticos en condiciones controladas o semi controlados, donde la calidad del agua es esencial para el desarrollo de las especies. Los parámetros que se toman en cuenta para determinar la calidad del agua son: temperatura, turbidez, transparencia, oxígeno disuelto, dióxido de carbono, $\mathrm{pH}$, alcalinidad, dureza, amonio, nitritos NO2, nitratos $\mathrm{NO} 3$.

Entre las fases de la producción de alevinos de Gamitana luego del desove, la etapa de mayor pertinencia se da durante los diez primeros días de vida (Curonisy Velarde et al., 2018). Por lo tanto, existe la necesidad de cuidados especiales para minimizar la mortalidad que actualmente en algunos casos sobre pasa el $50 \%$ dado a los cambios bruscos de los parámetros físico-químicos del agua como la temperatura, oxígeno, $\mathrm{pH}, \mathrm{NH} 4$ y $\mathrm{CO} 2$.

En el laboratorio de producción de alevinos de la Estación Pesquera Ahuashiyacu (EPA), espacio geográfico del presente estudio, se ha identificado la inexistencia de control de los parámetros físico-químicos del agua, por el contrario, en los estanques pretéritos se evidenciaron algunas mediciones de las cuales se deduce que el $71.6 \%$ y $16.25 \%$ de los datos registrados en relación a la temperatura y $\mathrm{pH}$ estuvieron fuera de los rangos permisibles.

De este modo, la alta tasa de mortalidad de Gamitana, está relacionada directamente con el monitoreo y control permanente de los parámetros físico-químicos del agua, además con la manipulación durante el desarrollo embrionario.

Bajo este panorama, el artículo busca determinar como la construcción de un sistema automatizado de control de parámetros físicos-químicos del agua reduce la tasa de mortalidad del Colossoma macropomum en los diez primeros días de vida en el proceso de reproducción artificial. 


\section{Materiales y métodos}

El estudio se realizó en el periodo agosto-diciembre del 2015, en el laboratorio de reproducción artificial de la EPA, ciudad de Tarapoto departamento de San Martín, con una ubicación geográfica de latitud: -6.48778 y longitud: $-76.35976^{\circ} 29^{\prime} 16^{\prime \prime}$ Sur, $76^{\circ} 21^{\prime} 35^{\prime \prime}$ Oeste. Para el experimento se utilizaron los reproductores de la especie Gamitana (Colossoma macropomum) y los materiales electrónicos descritos a continuación (Tabla I):

Tabla I. Materiales electrónicos utilizados

\begin{tabular}{|c|c|}
\hline Material & Descripción \\
\hline $\begin{array}{c}\text { Incubadoras del tipo } \\
\text { Woynarovich }\end{array}$ & $\begin{array}{c}\text { Funcionan con abastecimiento constante de agua, a fin de } \\
\text { mantener los huevos en movimiento ya que de otro modo se } \\
\text { adhieren entre si y mueren. }\end{array}$ \\
\hline $\begin{array}{c}\text { Plataforma Arduino } \\
\text { Uno Rev3 }\end{array}$ & $\begin{array}{r}\text { Placa electrónica basada en el chip de Atmel ATmega328. } \\
\text { Tiene I4 pines digitales de entrada / salida. }\end{array}$ \\
\hline XBEE & $\begin{array}{c}\text { Utiliza el protocolo IEEE 802.I5.4 para comunicaciones punto } \\
\text { a punto y punto a multipunto. Cuenta con una baja latencia de } \\
\text { transmisión, bajo consumo energético y largo alcance. }\end{array}$ \\
\hline $\begin{array}{c}\text { Módulo conversor de } \\
\text { voltaje de } \\
\text { viceversa }\end{array}$ & $\begin{array}{c}\text { Los conversores de voltaje bidireccional usados para la } \\
\text { transmisión y recepción de datos entre el microcontrolador } \\
\text { ATMEGA 328/I28AP con el módulo XBEE. }\end{array}$ \\
\hline $\begin{array}{c}\text { Sensor pH } \\
\text { Sensor Oxígeno } \\
\text { Disuelto }\end{array}$ & $\begin{array}{c}\text { La Sonda de pH (potencial de hidrógeno) mide la actividad de } \\
\text { los iones de hidrógeno en un líquido. }\end{array}$ \\
\hline $\begin{array}{c}\text { Una sonda galvánica de oxígeno disuelto consta de una } \\
\text { membrana de PTFE, un ánodo bañado en un electrolito y un } \\
\text { cátodo. }\end{array}$ \\
\hline $\begin{array}{c}\text { Temsor de } \\
\text { El DSI8B20 es un sensor digital de temperatura que utiliza el } \\
\text { protocolo I-Wire para comunicarse, este protocolo necesita } \\
\text { solo un pin de datos para comunicarse y permite conectar más } \\
\text { densmo bus. }\end{array}$ \\
\hline
\end{tabular}

Asimismo, se emplearon los siguientes componente electrónicos: Microcontrolador ATMEGA 328P-UP (incluye Bootloader); cristal de cuarzo de $16 \mathrm{MHz}$; socket para circuito integrado 28 pines; memoria EEPROM 24LC256; regulador de voltaje de 5V 5.55; regulador de voltaje de $3.3 \vee 5.55$; circuito impreso fibra de vidrio doble cara metalizado $10 \mathrm{~cm} \times 1 \mathrm{I} \mathrm{cm}$ aprox; antena WiFi de $2.4 \mathrm{GHz}$ con codo de $90^{\circ}$; caja de plástico según medida Fuente de poder de I Amp $12 \mathrm{~V}$; chip conversor USB serial; módulo de tres conversores de voltaje bidireccional de $3.3 \mathrm{~V}$ a $5 \mathrm{~V}$ y de $5 \mathrm{~V}$ a $3.3 \mathrm{~V}$; adaptador conversor USB y serial.

Respecto a la metodología empleada, se utilizó el método descriptivo experimental basado en tres componentes:

\subsection{Análisis del proceso de reproducción artificial}

Este componente se dividió en dos etapas: I) Identificación de proceso de producción, que consistió en indagar los procesos productivos de la reproducción artificial en la estación pesquera, para lo cual se realizó la investigación bibliográfica, consulta a los expertos de la estación pesquera Ahuashiyacu y trabajo de campo en la estación; y 2) Análisis de procesos, una vez reconocido los procesos se analizaron los procedimientos y se identificaron los principales parámetros de la calidad del agua de las incubadoras para el diseño de los instrumentos 
tecnológico para un mejor monitoreo y control de la producción de alevines en condiciones de laboratorio.

\subsection{Diseño e implementación del sistema de medición y control de los parámetros físico-químicos}

Se utilizó como elemento base el controlador Arduino. El nodo remoto tuvo por función adquirir los valores de los sensores de temperatura $(\mathrm{T})$, Potencial de Hidrógeno $(\mathrm{pH})$ y oxígeno disuelto (OD), así como también transmitir los datos de forma inalámbrica hacia el nodo concentrador, utilizando para ello el protocolo 802.15.4 o comercialmente denominado Zigbee. Dicho protocolo fue implementado en un transmisor/receptor XBEE. El nodo estuvo formado por los siguientes bloques (Figura I):

- Bloque de energía.

- Bloque de control y administración de datos.

- Bloque de adquisición de datos de los tres sensores (T. pH y OD).

- Bloque de transmisión inalámbrica, formado por el XBEE en modo Router/Terminal.

Figura I. Esquema electrónico del nodo remoto.
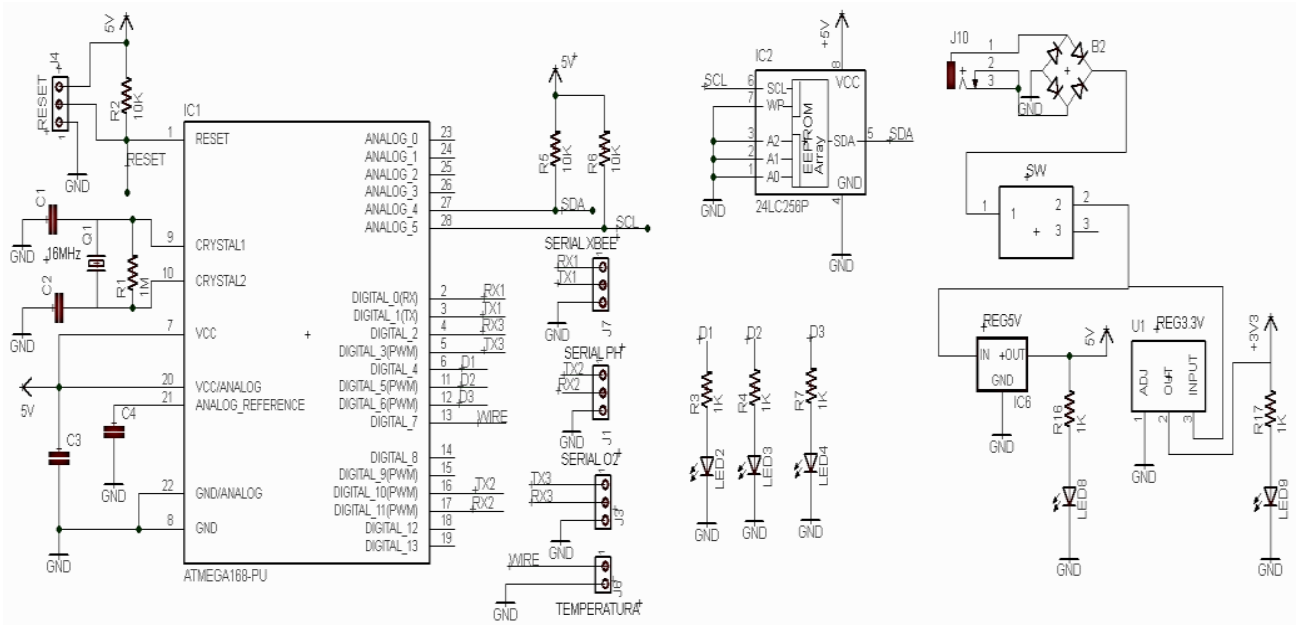

En relación al nodo concentrador, tuvo por función recibir y acumular los valores de los parámetros físicos y químicos provenientes de los nodos remotos. Este se conformó por los siguientes bloques (Figura 2):

- Bloque de energía.

- Bloque de almacenamiento de datos en memoria EEPROM.

- Bloque del reloj de tiempo real - RTC.

- Bloque de comunicación por serial-USB.

- Bloque de transmisión inalámbrica. 
Figura 2. Esquema electrónico del nodo concentrador.

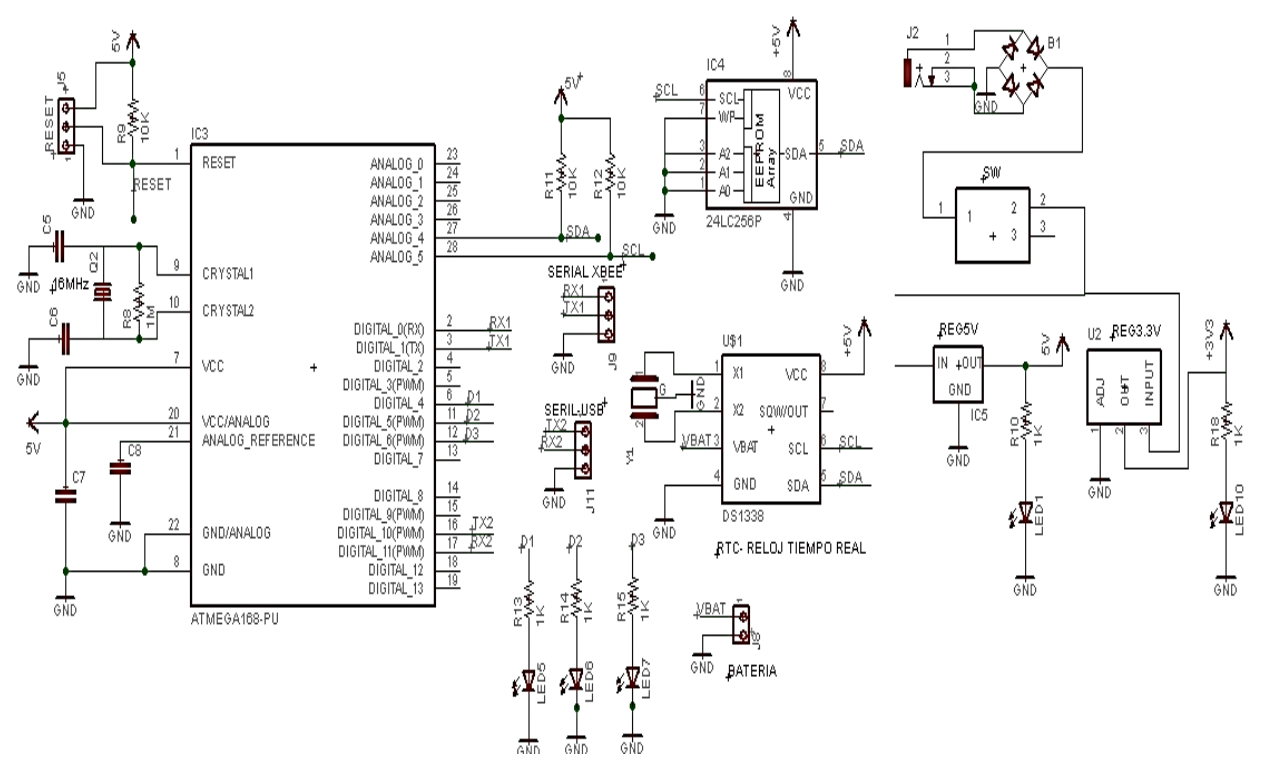

El desarrollo del software de control se realizó en $\mathrm{C}++$, lenguaje que soporta el micro controlador Arduino de la unidad concentradora de datos y de unidad remota o nodo de sensado. Posteriormente, se realizaron dos pruebas en el laboratorio de reproducción artificial, para finalmente implementar el sistema en las incubadoras.

\subsection{Medición de los parámetros físico-químicos del agua y tasa de mortalidad}

Con la implementación del sistema en el laboratorio de reproducción artificial de la EPA; se desarrolló el experimento en los laboratorios según los siguientes pasos: a) recolección de información, b) procesamiento de datos y c) validación de hipótesis.

Por otra parte, la investigación fue abordada por un Diseño Completamente Aleatorizado (DCA). Se realizaron tres replicas con dos tratamientos (grupo control y grupo experimental). Para el análisis de los datos se aplicó la estadística descriptiva e inferencial; además, se confeccionaron cuadros comparativos de estadígrafos con los resultados de los indicadores principales de la muestra antes y después, el mismo que permitió su medición de los cambios obtenidos en cuanto a su homogeneidad. Los datos contenidos fueron utilizados para la respectiva contrastación de la hipótesis, haciendo uso de la prueba estadística análisis de varianza, $T$ de student.

\section{Resultados y discusión}

De la revisión bibliográfica de Verdi-Olivares et al. (20l4), FONDEPES (2004), y la entrevista al director de la estación Biólogo Gilmer Raúl Montejo Sánchez, sobre los procesos productivos de la reproducción artificial en la estación pesquera, los protocolos de selección de reproductores para el tratamiento hormonal de Gamitana, con fines de inducción de la ovulación y desove, en condiciones controladas son coincidentes, obteniendo el siguiente diagrama de flujo de los procesos de reproducción artificial de larvas alevines (Figura 3). 
Figura 3. Flujograma del proceso de reproducción artificial de larvas de Gamitana.

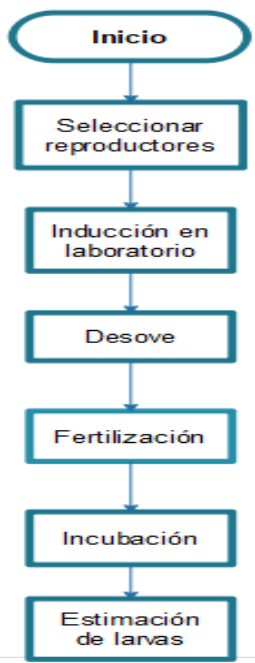

El sistema electrónico de monitoreo y control de los parámetros físicos-químicos se conformó por una red de cuatro módulos XBEE formada por un coordinador (Nodo Concentrador) y tres routers (Nodos remotos). En la figura 4, se muestra su esquema en bloques. Cabe resaltar que poseyó dos tanques de agua como sistema de abastecimiento cuyo flujo o caudal fue de aproximadamente I $000 \mathrm{l} / \mathrm{h}$.

Luego, dicho caudal de agua pasó por un sistema calentador cuya temperatura fue regulada de forma manual mediante un teclado, lográndose tener un control máximo de $2{ }^{\circ} \mathrm{C}$. Una vez temperada el agua, ingresó a 6 incubadoras con embriones de peces Gamitana, de los cuales tres se monitorearon electrónicamente por equipos remotos que incluyeron un microcontrolador ATMEGA 328/I28AP, un transmisor XBEE en modo ROUTER y sensores de temperatura, $\mathrm{pH}$ y oxígeno disuelto.

Para equilibrar los cambios de $\mathrm{pH}$ del agua generada por los nitritos y nitratos se integró en el sistema un equipo dosificador que a través de una electroválvula y electrobomba logró dosificar el cambio de $\mathrm{pH}$ usando sustancias basadas en fosfatos o ácido clorhídricos.

Figura 4. Esquema en bloques del sistema de monitoreo y control de incubadoras.

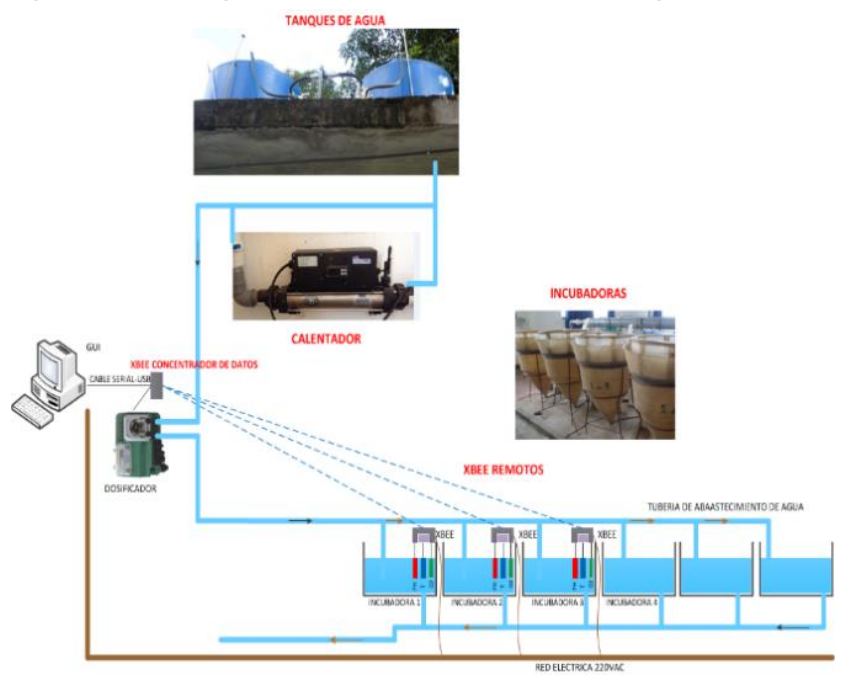


De acuerdo a Montesinos Navarro (2013) se ha podido evidenciar las principales características de la tecnología de comunicación inalámbrica ZigBee, entre las que se ha destacado su sencillez y eficacia para el despliegue, configuración y administración de redes adhoc auto-configurables de bajo consumo, gran alcance, adaptabilidad frente a condiciones impredecibles y elevado número de nodos. Estas prestaciones convierten a ZigBee en una tecnología inalámbrica superior a otras alternativas como Wi-Fi.

El diseño y construcción del sistema al igual que la propuesta de (Navarro Pérez et al., 2013) persiguen los mismos objetivos del monitoreo, sin embargo, el procedimiento fue distinto. Se seleccionaron y caracterizaron los sensores para medir temperatura del agua, $\mathrm{pH}$ y oxígeno disuelto en el agua, obteniéndose las funciones de transferencia de cada sensor. Se elaboraron tarjetas electrónicas para la adquisición y el procesamiento de la información usando tecnología basada en datalogger y puerto de comunicaciones USB, para luego en un estanque mediano simular condiciones artificiales de estanque, a diferencia de nuestra propuesta que se implementó "in situ", es decir en el laboratorio en condiciones reales, y se utilizó diferente tecnología.

La medición de los parámetros del agua se realizó en los diez días en intervalos de una hora cada uno, es decir, se obtuvieron 24 medidas por 10 días en tres replicas por dos tratamientos. Según el diseño propuesto, para mejor análisis de los datos obtenidos se calculó de la medida aritmética, desviación estándar y varianza, como se demuestran en la Tabla 2 y 3. En el grupo control con las condiciones habituales las mediciones de $\mathrm{pH}$, temperatura y oxígeno disuelto, en promedio obtenidos no tuvieron variación significativa.

Tabla 2. Mediciones obtenidas antes y después del grupo experimental

\begin{tabular}{|c|c|c|c|c|c|c|}
\hline & \multicolumn{3}{|c|}{ Medición antes } & \multicolumn{3}{c|}{ Medición después } \\
\hline $\mathrm{N}^{\circ}$ & $\mathrm{pH}$ & $\mathrm{T}$ & $\mathrm{OD}$ & $\mathrm{pH}$ & $\mathrm{T}$ & $\mathrm{OD}$ \\
\hline $\mathrm{I}$ & 6.999 & 25.066 & 6.23 & 7.699 & 26.115 & 8.132 \\
\hline 2 & 7.199 & 25.010 & 6.792 & 7.806 & 26.223 & 8.225 \\
\hline 3 & 6.732 & 24.825 & 7.003 & 7.705 & $26.22 \mathrm{I}$ & 8.250 \\
\hline 4 & 5.997 & 25.246 & 6.954 & 7.701 & 26.253 & 8.407 \\
\hline 5 & 7.536 & 24.828 & 6.883 & 7.807 & $26.13 \mathrm{I}$ & 8.207 \\
\hline 6 & 7.059 & 24.568 & 7.000 & 7.780 & 25.928 & 8.191 \\
\hline 7 & 7.404 & 24.073 & 6.057 & 7.689 & 25.586 & 8.258 \\
\hline 8 & 7.516 & $24.71 \mathrm{I}$ & 5.902 & 7.576 & 25.624 & 8.208 \\
\hline 9 & 7.422 & 24.739 & 5.691 & 7.226 & 25.663 & $8.17 \mathrm{I}$ \\
\hline $\mathrm{I0}$ & 7.182 & 24.522 & 6.187 & 7.352 & 25.647 & 8.216 \\
\hline Media & 7.015 & 24.759 & 6.4699 & 7.634 & 25.939 & 8.233 \\
\hline Desv. Est. & 0.464 & 0.328 & 0.507 & 0.196 & 0.281 & 0.074 \\
\hline Varianza & 0.216 & 0.108 & 0.257 & 0.038 & 0.079 & 0.005 \\
\hline
\end{tabular}


Tabla 3. Mediciones obtenidas antes y después del grupo control

\begin{tabular}{|c|c|c|c|c|c|c|}
\hline & \multicolumn{3}{|c|}{ Medición antes } & \multicolumn{3}{c|}{ Medición después } \\
\hline $\mathrm{N}^{\circ}$ & $\mathrm{pH}$ & $\mathrm{T}$ & $\mathrm{OD}$ & $\mathrm{pH}$ & $\mathrm{T}$ & $\mathrm{OD}$ \\
\hline $\mathrm{I}$ & 6.999 & 25.066 & 6.23 & 6.999 & 25.066 & 6.23 \\
\hline 2 & 7.199 & 25.010 & 6.792 & 7.199 & 25.010 & 6.792 \\
\hline 3 & 6.732 & 24.825 & 7.003 & 6.732 & 24.825 & 7.003 \\
\hline 4 & 5.997 & 25.246 & 6.954 & 5.997 & 25.246 & 6.954 \\
\hline 5 & 7.536 & 24.828 & 6.883 & 7.536 & 24.822 & 6.883 \\
\hline 6 & 7.059 & 24.568 & 7.00 & 7.059 & 24.568 & 7.00 \\
\hline 7 & 7.404 & 24.073 & 6.057 & 7.404 & 24.073 & 6.057 \\
\hline 8 & 7.516 & $24.71 \mathrm{I}$ & 5.902 & 7.516 & $24.71 \mathrm{I}$ & 5.902 \\
\hline 9 & 7.422 & 24.739 & $5.69 \mathrm{I}$ & 7.422 & 24.739 & $5.69 \mathrm{I}$ \\
\hline $\mathrm{I0}$ & 7.182 & 24.522 & 6.187 & 7.182 & 24.522 & 6.187 \\
\hline Media & 7.105 & 24.759 & 6.4699 & 7.105 & 24.759 & 6.469 \\
\hline Desv. Est. & 0.464 & 0.328 & 0.507 & 0.464 & 0.328 & 0.507 \\
\hline Varianza & 0.216 & 0.108 & 0.257 & 0.216 & 0.108 & 0.257 \\
\hline
\end{tabular}

En la EPA se tuvo seis incubadoras de $210 \mathrm{I}$, para la presente investigación se decidió ocupar el total de incubadoras, tres para el grupo experimental y tres para el grupo control. Las reproductoras existentes presentaron un peso entre $4 \mathrm{Kg}$. a $5 \mathrm{Kg}$. Dependiendo de la calidad de alimento que han recibido los reproductores se obtiene en promedio 100000 óvulos por kilogramo. Los datos obtenidos de las tres replicas con dos tratamientos, se muestran en las Tablas 4, 5 y 6 siguientes:

Tabla 4. Replicas en condiciones normales

\begin{tabular}{|c|c|c|c|c|}
\hline $\mathbf{N}^{\circ}$ & Cantidad & Huevos & $\mathbf{N}^{\circ}$ & $\mathbf{N}^{\circ}$ \\
\hline Replicas & Óvulos & Fecundados & Larvas & Pos Larvas \\
\hline I & 240000 & 192000 & 134400 & 67200 \\
\hline 2 & 240000 & 180000 & 117000 & 58500 \\
\hline 3 & 240000 & 187200 & 163200 & 81600 \\
\hline
\end{tabular}

Tabla 5. Replicas en condiciones controladas

\begin{tabular}{|c|c|c|c|c|}
\hline $\mathbf{N}^{\circ}$ & Cantidad & Huevos & $\mathbf{N}^{\circ}$ & $\mathbf{N}^{\circ}$ \\
\hline Replicas & Ovulos & Fecundados & Larvas & Pos Larvas \\
\hline I & 240000 & I57 440 & I2I9 I00 & 77460 \\
\hline 2 & 240000 & 192000 & I57 440 & I I0 208 \\
\hline 3 & 240000 & 199200 & 159360 & 103584 \\
\hline
\end{tabular}

Tabla 6. Número de pos larvas en el grupo de control y grupo experimental

\begin{tabular}{|c|c|c|}
\hline & Grupo control & Grupo experimental \\
\hline $\mathrm{I}$ & 67200 & 77460 \\
\hline 2 & 58500 & 110208 \\
\hline 3 & 81600 & 103584 \\
\hline
\end{tabular}

La Tabla 7 muestra el análisis correspondiente a la medición $\left(\mathrm{O}_{2}-\mathrm{O}_{4}\right)$ para la comparación de la producción de pos larvas en condiciones normales y en condiciones controladas. Al aplicar la prueba $\mathrm{T}$ student, el valor de $\mathrm{t}$ calculada $(\mathrm{Tc}=2.268)$ fue mayor al valor de $\mathrm{t}$ tabulada $(\mathrm{Tt}=$ 2.219), en la prueba unilateral de cola a la derecha, ubicándose en la región de rechazo. Por consiguiente, se rechazó la hipótesis nula, es decir, los resultados de la producción de pos larvas en el post test del grupo experimental son significativamente mayores a los del post test del 
grupo control. Según este análisis la tasa de mortalidad de la especie Gamitana durante los diez primeros días de vida en reproducción artificial, disminuyó mediante el control automatizado de los parámetros físico-químicos del agua.

Tabla 7. Influencia del sistema automatizado en la supervivencia de pos larvas

\begin{tabular}{|c|c|c|c|c|}
\hline \multicolumn{5}{|c|}{ Comparación entre grupos pre y post test del grupo experimental y grupo control } \\
\hline Hipótesis & Nivel de significancia & T calculada & T tabulada & Decisión \\
\hline $\begin{array}{c}\mathrm{H}_{\mathrm{O}}: \mu 02=\mu \mathrm{I} \\
\mathrm{H}: \mathrm{I}: \mu \mathrm{O}_{2}>\mu \mathrm{I}\end{array}$ & $\mathbf{5} \%$ & 2.919 & 2.268 & $\begin{array}{c}\text { Rechaza Ho: } \\
\mu 02=\mu 04\end{array}$ \\
\hline
\end{tabular}

La veracidad de la hipótesis planteada permite inferir que es factible diseñar un prototipo capaz de registrar mediciones de los parámetros de la calidad del agua en un estanque tal cual lo sostienen los autores Rivera Herrera \& Yepez Aroca (2015). Asimismo, se ha podido evidenciar que las variables físicas como son la temperatura, el $\mathrm{pH}$ y el oxígeno disuelto que intervienen en la tasa de mortalidad de los alevines de Gamitan, y que son perceptibles de medición por sensores, presentan una gran precisión en la medición de cada variable de acuerdo a los parámetros en condiciones de temperatura ambiente (Navarro Pérez et al., 2013).

\section{Conclusiones}

Se diseñó un sistema automatizado partiendo del análisis de los procesos de reproducción artificial, el sistema se constituyó por sensores, actuadores, nodo concentrador de sensores con base Arduino. La tasa de mortalidad en condiciones normales fue de $71.2 \%$, mientras que con el uso del sistema de control la tasa pasó a $59.5 \%$, es decir, con el uso del sistema se ha disminuido la tasa de mortalidad en un $21.7 \%$.

Asimismo, se han medido los principales parámetros físicos químicos del agua de forma periódica y continua con el uso de sensores del sistema automatizado de los cuales se obtuvo que las mediciones de $\mathrm{pH}$ obtenidos presentaron diferencias significativas en el cálculo de la desviación estándar; en cuanto a la temperatura los datos obtenidos estuvieron por debajo del rango permitido, por lo que el actuador lo estabilizó en $25^{\circ} \mathrm{C}$; y el oxígeno disuelto se encontró sin variaciones significativas.

\section{Referencias bibliográficas}

Alvan-Aguilar, M. A., Boullosa L., M. C., Valderrama C., S. A., Rodríguez Ch., L. A., Ruiz T., K. M., Ismiño O., R. A., \& Chu-Koo, F. W. (202I). Evaluación de parámetros reproductivos de Colossoma macropomum "gamitana", en el Centro de Investigaciones Fernando Alcántara Bocanegra CIFAB-IIAP, Loreto, Perú. Boletin Instituto Del Mar Del Perú, 35(I), |34-| 42. https://revistas.imarpe.gob.pe/index.php/boletin/article/view/297

Curonisy Velarde, Y., Ignacio Pastén, J., \& Chong Chong, M. (2018). Proyecto de comercialización de la gamitana en Lima, Perú. Industrial Data, 2 I (2), 73-80. https://doi.org//0.1538I/idata.v2Ii2.15605

DIREPRO. (2014). Plan Regional de Acuicultura de San Martín 2014 -2023. Ordenanza Regional $N^{\circ}$ 024-20 I4-GRSM/CR. Dirección Regional de la Producción de San Martín.

FAO. (2016). El estado mundial de la pesca y la acuicultura 2016. Contribución a la seguridad 
alimentaria y la nutrición para todos. Roma. https://www.fao.org/3/i5555s/i5555s.pdf

FONDEPES. (2004). Manual de Cultivo de Gamitana. Fondo Nacional de Desarrollo Pesquero. http://www2.produce.gob.pe/RepositorioAPS/3/jer/ACUISUBMENU4/manual_gamitana.P df

Montesinos Navarro, J. S. (2013). Red de sensores auto-configurable mediante tecnología ZigBee y Arduino con monitorización por aplicación Android [Universidad Politécnica de Cartagena]. http://hdl.handle.net/ I03 /7/3678

Navarro Pérez, Á. A., Padilla Bejarano, J. B., \& Prías Barragán, J. J. (20I3). Construcción de un Sistema de Instrumentación para la Medición de la Temperatura, $\mathrm{pH}$ y Oxígeno Disuelto presentes en la Piscicultura bajo Condiciones de Estanque Artificial. Scientia Et Technica, I8(2), 40 I-408. https://www.redalyc.org/articulo.oa?id=84929|530I7

Rivera Herrera, D. I., \& Yepez Aroca, E. A. (2015). Diseño e implementación de un prototipo para la medición de calidad del agua y control de la oxigenación en forma remota orientado a la producción acuícola. [Universidad Politécnica Salesiana]. http://dspace.ups.edu.ec/handle//23456789// 0328

Sánchez Calle, J. E., Valles Coral, M. Á., \& Gonzales Sánchez, P. A. (202I). Políticas promovedoras de la tecnificación y su efecto en la productividad acuícola. Ciencia \& Tecnología Agropecuaria, 22(3), e2 100. https://doi.org/10.21930/rcta.vol22_num3_art:2100

Verdi-Olivares, L., Alcántara-Bocanegra, F., Rodríguez-Chu, L., Chu-Koo, F., Ramírez-Arrarte, P., \& Tello-Martín, S. (20I4). Validación del Protocolo de Reproducción de Colossoma macropomum, Piaractus brachypomus y Prochilodus nigricans en Condiciones Controladas. Ciencia Amazónica (lquitos), 4(I), 54-59. https://doi.org/I0.22386/ca.v4il .68

\section{Financiamiento}

Universidad Nacional de San Martín mediante Resolución N I38-20I5-UNSM/CU-R/NLU.

\section{Conflicto de intereses}

El artículo no presenta conflicto de intereses.

\section{Contribución de autores}

García-Castro, Juan y Ascón-Dionicio, Gilberto: Investigadores y redactores del manuscrito. Ambos participaron en la conceptualización del estudio, aplicación de experimentos y procesamiento estadístico. 